\title{
Impedance of a planar solenoid with a thin magnetic core
}

\author{
Behzad Rejaei ${ }^{\mathrm{a})}$ and Marina Vroubel \\ High Frequency Technology and Components Group, Faculty of Electrical Engineering, Mathematics, \\ and Computer Science, Delft University of Technology, Feldmannweg 17, 2628 CT Delft, The Netherlands
}

(Received 12 September 2006; accepted 31 January 2007; published online 10 April 2007)

\begin{abstract}
The high-frequency impedance of a planar solenoid with a thin magnetic core is theoretically investigated using the magnetostatic Green's function formalism. It is shown that the electrical behavior of the solenoid depends on how the magnetic field induced by the current-carrying coil is coupled to the different magnetostatic modes of the core. The magnetic response of the core in each mode is determined by an effective susceptibility matrix which depends on exact, but mode-dependent demagnetization factors. Those factors determine the frequency of the magnetostatic excitations of the core, manifested as resonances in the impedance of the solenoid. Using the formalism developed, the effect of the core width and magnetic loss on the impedance of the device is studied. (C) 2007 American Institute of Physics. [DOI: 10.1063/1.2715760]
\end{abstract}

\section{INTRODUCTION}

The need for miniaturized components for radio frequency (rf) and microwave integrated circuits has led to a surge of interest in devices utilizing thin, high-permeability, magnetic films. In particular, integrated planar inductors with thin magnetic cores ${ }^{1}$ have attracted much attention, in view of their potential application in rf filters, resonators, and matching circuits. Spiral, ${ }^{2-4}$ sandwiched stripe, ${ }^{5}$ and planar solenoid $^{6,7}$ designs using thin magnetic films have already been proposed and studied. Compared to spirals, sandwiched stripes and planar solenoids benefit more from the incorporation of the magnetic film, due to the favorable direction of the magnetic rf field with respect to the orientation of the magnetization in the core. ${ }^{8}$

For the design and analysis of magnetic inductors, knowledge of the rf susceptibility of the magnetic core used is essential. The susceptibility of a magnetic element with a general nonellipsoidal shape is usually described in terms of its averaged demagnetization factors, ${ }^{9-11}$ in analogy with the description of the uniform precessional mode of a magnetic ellipsoid. ${ }^{12}$ The demagnetization factors are determined by averaging the demagnetization field generated by a uniform magnetization profile in the nonellipsoidal sample. ${ }^{13}$ This approach, however, cannot be justified in miniature magnetic inductors where the field generated by the current-carrying wires is highly nonuniform. Furthermore, it is not obvious how one should take into account the effect of the magnetostatic excitations ${ }^{14-16}$ of the magnetic core on the electrical characteristics of the inductor.

In this paper we present a theoretical analysis of the high-frequency impedance of a planar solenoid with a core built from a thin, long magnetic stripe. ${ }^{6,7}$ Using the thin-film approximation of the magnetostatic Green's function formalism, ${ }^{18}$ the high-frequency behavior of the core is described in terms of its magnetostatic modes. It is shown that the magnetic response of the core in each mode is determined by an effective susceptibility matrix. The latter is ex-

${ }^{a)}$ Electronic mail: b.rejaei@ewi.tudelft.nl pressed in terms of exact, but mode-dependent demagnetization factors. The impedance of the solenoid is found by evaluating the degree of coupling of the magnetic field of the current-carrying coil to each mode. The magnetostatic excitations of the core are manifested as resonances in the impedance of the solenoid, with the resonance frequencies determined by the mode-dependent demagnetization factors. Using the formalism developed, examples are given to illustrate the effect of the core width and magnetic loss on the impedance of the device.

This paper is organized as follows. In Sec. II the thinfilm approximation is used to determine the high-frequency response of a magnetic stripe. The results are used in Sec. III to calculate the impedance of a planar solenoid. In Sec. IV we employ the formalism developed to analyze the electrical characteristics of several solenoids. The paper is concluded in Sec. V.

\section{SUSCEPTIBILITY OF A THIN MAGNETIC STRIPE}

Figure 1 shows a planar solenoid consisting of a planar coil enclosing a thin, long magnetic stripe. The conductors comprising the coil are electrically isolated from the magnetic core by dielectric layers. The magnetic stripe is saturated in the $z$ direction with the uniform static magnetization $M_{s} \hat{z}$, under the influence of an effective static field $H_{a} \hat{z}$.

A rf current flowing through the windings of the coil generates a time harmonic rf field, which we call the external field. For simplicity, we assume the length of the solenoid to be much larger than its width and neglect the variations in the $z$ direction. The $\mathrm{rf}$ field of the coil causes the forced oscillation of the magnetization inside the stripe. Under small signal conditions, where the magnitude of the induced rf magnetization $\boldsymbol{m}$ is much smaller than $M_{s}$, the longitudinal component of the rf magnetization can be neglected, i.e., $\boldsymbol{m}$ $=m_{x} \hat{\boldsymbol{x}}+m_{y} \hat{\boldsymbol{y}}^{17}$

In order to analyze the response of the rf magnetization inside the stripe to the field of the coil, we use the tensorial 

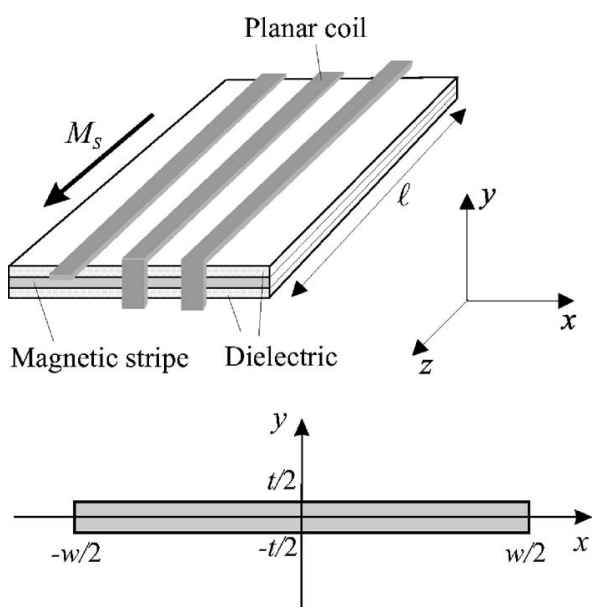

FIG. 1. Top: A planar solenoid built from a planar coil enclosing a thin magnetic stripe. The coil is electrically isolated from the stripe by dielectric layers. The stripe is assumed to have a uniform static magnetization $M_{s}$ in the $+z$ direction. Bottom: The cross section of the magnetic stripe in the $x-y$ plane.

Green's functions formalism. ${ }^{18,19}$ The derivation is outlined below in several steps. At each point inside the magnetic stripe one has

$$
\boldsymbol{m}=\overline{\bar{\chi}} \cdot \boldsymbol{h},
$$

where

$$
\overline{\bar{\chi}}=\frac{\omega_{M}}{\omega_{H}^{2}-\omega^{2}}\left[\begin{array}{cc}
\omega_{H} & i \omega \\
-i \omega & \omega_{H}
\end{array}\right]
$$

is the $2 \times 2$ susceptibility tensor in which $\omega$ is the (angular) frequency of the rf field, and $\omega_{M}=\gamma M_{s}$ and $\omega_{H}=\gamma H_{a}$ with $\gamma$ as the gyromagnetic constant. ${ }^{17}$ In Eq. (1) $\boldsymbol{h}$ is the total magnetic field, which in the magnetostatic approximation of the Maxwell equations can be written as

$$
\boldsymbol{h}=\boldsymbol{h}_{e}+\boldsymbol{h}_{m},
$$

where $\boldsymbol{h}_{e}=h_{e, x} \hat{\boldsymbol{x}}+h_{e, y} \hat{\boldsymbol{y}}$ is the transversal part of the external rf field, and $\boldsymbol{h}_{m}$ is the transversal demagnetization field given by

$$
\begin{aligned}
& \boldsymbol{h}_{m}(\boldsymbol{r})=-\nabla \int_{S} \nabla^{\prime} \mathcal{G}\left(\boldsymbol{r}, \boldsymbol{r}^{\prime}\right) \cdot \boldsymbol{m}\left(\boldsymbol{r}^{\prime}\right) d S^{\prime}, \\
& \mathcal{G}\left(\boldsymbol{r}, \boldsymbol{r}^{\prime}\right)=-\frac{1}{2 \pi} \ln \left|\boldsymbol{r}-\boldsymbol{r}^{\prime}\right|
\end{aligned}
$$

Here $\boldsymbol{r}=(x, y), \nabla=\left(\partial_{x}, \partial_{y}\right)$, and $S$ is the cross section of the magnetic stripe in the $x-y$ plane.

In practical solenoids with thin-film cores, the spatial variation of the rf field generated by the coil (and the induced rf magnetization) is negligible over distances comparable to the magnetic film thickness. Thus, the field quantities can be replaced by their values averaged over the thickness $t$ of the magnetic film,

$$
\boldsymbol{m}(\boldsymbol{r}) \rightarrow \widetilde{\boldsymbol{m}}(x)=\frac{1}{t} \int_{-t / 2}^{t / 2} \boldsymbol{m}(\boldsymbol{r}) d y,
$$

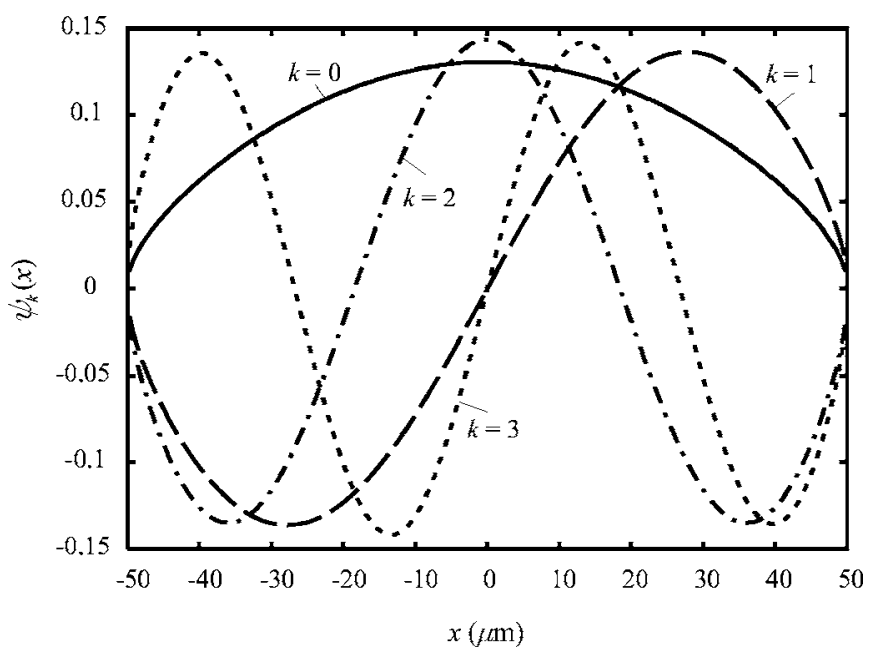

FIG. 2. The first four eigenfunctions $\psi_{k}(x)(k=0,1,2,3)$ for a magnetic stripe with a width of $100 \mu \mathrm{m}$ and a thickness of $0.2 \mu \mathrm{m}$. The corresponding eigenvalues are $\lambda_{0}=-0.99768, \lambda_{1}=-0.99447, \lambda_{2}=-0.99131$, and $\lambda_{3}=$ -0.98811 . The results were obtained by numerically solving Eq. (8).

$$
\boldsymbol{h}_{e, m}(\boldsymbol{r}) \rightarrow \widetilde{\boldsymbol{h}}_{e, m}(x)=\frac{1}{t} \int_{-t / 2}^{t / 2} \boldsymbol{h}_{e, m}(\boldsymbol{r}) d y
$$

Upon averaging Eq. (4a) and using Eqs. (1)-(3), one obtains the following integral equation for the average magnetization,

$$
\overline{\overline{\boldsymbol{Q}}} \cdot \widetilde{\boldsymbol{m}}(x)+\overline{\overline{\boldsymbol{\Sigma}}}_{3} \cdot \int_{-w / 2}^{w / 2} G\left(x, x^{\prime}\right) \widetilde{\boldsymbol{m}}\left(x^{\prime}\right) d x^{\prime}=\widetilde{\boldsymbol{h}}_{e}(x),
$$

where $w$ is the width of the stripe (Fig. 1), and

$$
\begin{aligned}
& \overline{\bar{Q}}=\frac{1}{\omega_{M}}\left[\begin{array}{cc}
\omega_{H}+\omega_{M} & -i \omega \\
i \omega & \omega_{H}
\end{array}\right], \\
& \overline{\overline{\mathbf{\Sigma}}}_{3}=\left[\begin{array}{cc}
1 & 0 \\
0 & -1
\end{array}\right], \\
& G\left(x, x^{\prime}\right)=\frac{1}{2 \pi t} \ln \left[\frac{\left(x-x^{\prime}\right)^{2}}{\left(x-x^{\prime}\right)^{2}+t^{2}}\right] .
\end{aligned}
$$

The dynamic response of the magnetic stripe, i.e., its susceptibility, can be in principle found by directly solving Eq. (6) for a given external field. Instead, however, we use this equation to express the susceptibility of the magnetic stripe in terms of its magnetostatic eigenmodes. ${ }^{20}$ The latter are found by the solution of the equation ${ }^{21}$

$$
\int_{-w / 2}^{w / 2} G\left(x, x^{\prime}\right) \psi_{k}\left(x^{\prime}\right) d x^{\prime}=\lambda_{k} \psi_{k}(x)
$$

Figure 2 shows the first four normalized eigenmodes $\psi_{k}(x)$ of a $100-\mu \mathrm{m}$-wide and $0.2-\mu \mathrm{m}$-thick stripe, obtained by the numerical solution of Eq. (8). ${ }^{23}$ Note that $\psi_{k}(x)$ is an even/odd function of $x$ for even/odd values of the integer $k$.

The solution of Eq. (6) can now be found by expanding the magnetization in the eigenmodes of the stripe,

$$
\widetilde{\boldsymbol{m}}(x)=\sum_{k=0}^{\infty} \widetilde{\boldsymbol{m}}^{k} \psi_{k}(x)
$$




$$
\widetilde{\boldsymbol{m}}^{k}=\int_{-w / 2}^{w / 2} \widetilde{\boldsymbol{m}}(x) \psi_{k}(x) d x
$$

where $\widetilde{\boldsymbol{m}}^{k}$ is a constant vector. By substituting Eq. (9a) into Eq. (6), using Eq. (8) and the orthonormality of the eigenmodes, it can be shown that

$$
\begin{aligned}
& \widetilde{\boldsymbol{m}}^{k}=\overline{\overline{\boldsymbol{\chi}}}^{k} \cdot \widetilde{\boldsymbol{h}}_{e}^{k}, \\
& \tilde{\boldsymbol{h}}_{e}^{k}=\int_{-w / 2}^{w / 2} \tilde{\boldsymbol{h}}_{e}(x) \psi_{k}(x) d x .
\end{aligned}
$$

Here $\widetilde{\boldsymbol{h}}_{e}^{k}$ is the amplitude of the external field in the $k$ th mode, and $\overline{\boldsymbol{\chi}}^{k}$ is a mode-dependent, effective susceptibility matrix given by

$$
\begin{aligned}
\overline{\overline{\boldsymbol{\chi}}}^{k} & =\left[\begin{array}{cc}
\chi_{x x}^{k} & i \chi_{a}^{k} \\
-i \chi_{a}^{k} & \chi_{y y}^{k}
\end{array}\right] \\
& =\frac{\omega_{M}}{\omega_{k}^{2}-\omega^{2}}\left[\begin{array}{cc}
\omega_{H}+N_{y}^{k} \omega_{M} & i \omega \\
-i \omega & \omega_{H}+N_{x}^{k} \omega_{M}
\end{array}\right],
\end{aligned}
$$

where $N_{x}^{k}=1+\lambda_{k}, N_{y}^{k}=-\lambda_{k}$, and

$$
\omega_{k}^{2}=\left(\omega_{H}+N_{x}^{k} \omega_{M}\right)\left(\omega_{H}+N_{y}^{k} \omega_{M}\right) .
$$

In analogy with the effective susceptibility matrix for the uniform precessional mode of a magnetic ellipsoid, one can interpret $N_{x}^{k}$ and $N_{y}^{k}$ as demagnetization factors in the $x$ and $y$ directions, respectively, incorporating the effect of the demagnetization field. Those factors determine $\omega_{k}$, the magnetostatic resonance frequency of the stripe in the $k$ th mode. The existence of modal susceptibility matrices has already been discussed in Ref. 22 for magnetic samples of general shape.

In practical cases one cannot neglect the effect of magnetic relaxation processes on the motion of the magnetization inside a magnetic sample. The resulting dissipation can be phenomenologically taken into account by making the replacement $\omega_{H} \rightarrow \omega_{H}-i \alpha \omega$ in Eqs. (11) and (12), where $\alpha$ is the Gilbert damping constant. ${ }^{17}$ Figure 3 shows the frequency dependence of the in-plane susceptibility $\chi_{x x}^{k}(k$ $=0,1,2,3)$ of a $100-\mu \mathrm{m}$-wide and $0.2-\mu \mathrm{m}$-thick stripe, with $M_{s}=1 \mathrm{~T}, H_{a}=1 \mathrm{Oe}$, and $\alpha=0.01$. For each mode, $\operatorname{Im}\left[\chi_{x x}^{k}\right]$ peaks at $\omega=\omega_{k}$, the magnetostatic resonance frequency. Note that the frequency linewidth $\Delta \omega$ of each resonance (defined as the distance between the two frequencies at which $\operatorname{Im}\left[\chi_{x x}^{k}\right]$ acquires half its peak value) is almost mode independent. In fact, it can be shown that $\Delta \omega \sim \alpha \omega_{M}$ if $\alpha \ll 1$. $^{24}$.

\section{IMPEDANCE OF A SOLENOID WITH A THIN MAGNETIC CORE}

Having provided an analysis of the rf susceptibility of a thin magnetic stripe, we now proceed to calculate the impedance of the solenoid. This is achieved by viewing the coil as an impressed current source, generating an external magnetic field $\boldsymbol{h}_{e}$. Thus, we assume that the distribution of the current inside the windings of the coil is not affected by the presence of the magnetic stripe. Now consider the total complex magnetic power delivered to the solenoid,
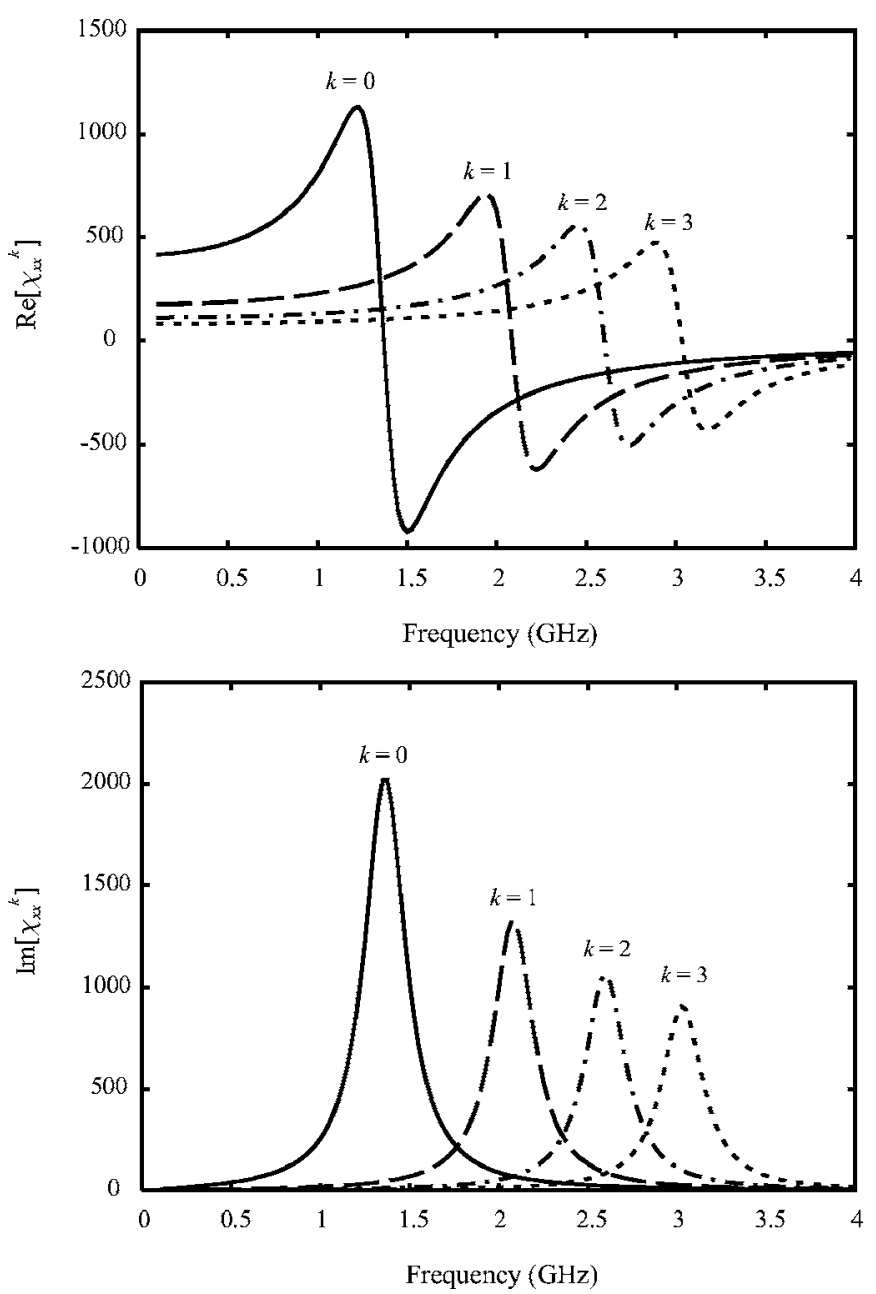

FIG. 3. Real and imaginary parts of the in-plane susceptibility $\chi_{x x}^{k}$ of a magnetic stripe as function of frequency for the first four modes $(k$ $=0,1,2,3)$. The stripe is $100-\mu \mathrm{m}$-wide and $0.2-\mu \mathrm{m}$-thick, with $M_{s}=1 \mathrm{~T}$, $H_{a}=1 \mathrm{Oe}$, and $\alpha=0.01$. Modes with a larger $N_{x}^{k}=1+\lambda_{k}$ have a higher magnetostatic resonance frequency.

$$
P_{M}=\frac{i \omega \ell}{2} \int \boldsymbol{h}(\boldsymbol{r}) \cdot \boldsymbol{b}^{*}(\boldsymbol{r}) d S
$$

where $\ell$ is the length of the solenoid and $\boldsymbol{b}=\mu_{0}(\boldsymbol{h}+\boldsymbol{m})$ is the magnetic induction with $\mu_{0}$ the permeability of vacuum. The integration in Eq. (13) is carried out over the whole $x-y$ plane. The impedance of the solenoid can then be expressed as

$$
Z=\frac{2 P_{M}}{|I|^{2}}+R_{s},
$$

where $I$ is the total current flowing through the coil. The term $R_{s}$ has been included to account for the series resistance of the coil.

By substituting Eq. (3) into Eq. (13), exploiting the fact that the demagnetization field $\boldsymbol{h}_{m}$ is a gradient function [see Eq. (4a)], applying partial integration, and using the Maxwell equation $\nabla \cdot \mathbf{b}=0$, it can be shown that ${ }^{22}$ 


$$
P_{M}=\frac{i \omega \mu_{0} \ell}{2}\left[\int\left|\boldsymbol{h}_{e}(\boldsymbol{r})\right|^{2} d S+\int_{S} \boldsymbol{h}_{e}(\boldsymbol{r}) \cdot \boldsymbol{m}^{*}(\boldsymbol{r}) d S\right] .
$$

The second integration in Eq. (15) is performed over the cross section of the stripe only. From this equation, and the definition of the impedance $Z$, it follows that

$$
\begin{aligned}
& Z=R_{s}+Z_{0}+Z_{m}, \\
& Z_{0}=\frac{i \omega \mu_{0} \ell}{|I|^{2}} \int\left|\boldsymbol{h}_{e}(\boldsymbol{r})\right|^{2} d S, \\
& Z_{m}=\frac{i \omega \mu_{0} \ell}{|I|^{2}} \int_{S} \boldsymbol{h}_{e}(\boldsymbol{r}) \cdot \boldsymbol{m}^{*}(\boldsymbol{r}) d S .
\end{aligned}
$$

Note that $R_{s}+Z_{0}$ is the impedance of the coil in the absence of the magnetic core, while $Z_{m}$ is the extra impedance caused by the inclusion of the latter.

Upon using the average field quantities defined by Eq. (5), substituting Eq. (9a) into Eq. (16c), and using Eq. (10), one obtains

$$
\begin{aligned}
& Z_{m}=\sum_{k=0}^{\infty} Z_{k}, \\
& Z_{k}=\frac{i \omega \mu_{0} t \ell}{|I|^{2}}\left[\widetilde{\boldsymbol{h}}_{e}^{k} \cdot\left(\overline{\overline{\boldsymbol{\chi}}}^{k} \cdot \widetilde{\boldsymbol{h}}_{e}^{k}\right)^{*}\right] .
\end{aligned}
$$

Therefore, $Z_{m}$ equals the sum of the partial impedances $Z_{k}$, whose value depends on the amplitude $\widetilde{\mathbf{h}}_{e}^{k}$ of the (averaged) external field and the susceptibility matrix $\overline{\overline{\boldsymbol{\chi}}}^{k}$ in the corresponding magnetostatic mode. It is possible to further simplify Eq. (17b) by noting that in the magnetostatic limit of the Maxwell equations, where electromagnetic wave propagation effects are neglected, the $x$ - and $y$-components of the external field have the same phase everywhere. As a result, the nondiagonal elements of $\overline{\overline{\boldsymbol{\chi}}}^{k}$ are eliminated from the expression for $Z_{k}$, yielding

$$
Z_{k}=\frac{i \omega \mu_{0} t \ell}{|I|^{2}}\left(\chi_{x x}^{k}\left|\widetilde{h}_{e, x}^{k}\right|^{2}+\chi_{y y}^{k}\left|\widetilde{h}_{e, y}^{k}\right|^{2}\right)^{*}
$$

where $\widetilde{h}_{e, x}^{k}$ and $\widetilde{h}_{e, y}^{k}$ denote the $x$ - and $y$-components of the amplitude $\widetilde{\mathbf{h}}_{e}^{k}$, respectively.

\section{NUMERICAL RESULTS}

The overall behavior of the impedance of a solenoid as function of frequency depends on how the magnetic field induced by the coil is coupled to the different magnetostatic modes of the core, each with its specific susceptibility. In what follows we analyze the impedance of a few solenoids by using Eqs. (16a), (16b), (17a), and (18). In each case, the field $\mathbf{h}_{e}$ (and its average $\tilde{\mathbf{h}}_{e}$ ) is computed in the absence of the magnetic core, assuming a uniform distribution of current density across the conductors comprising the coil. The magnetostatic eigenvalues and eigenfunctions are calculated by the numerical solution of Eq. (8).

For our numerical experiments we consider a single-turn planar solenoid where the two isolating dielectric layers have
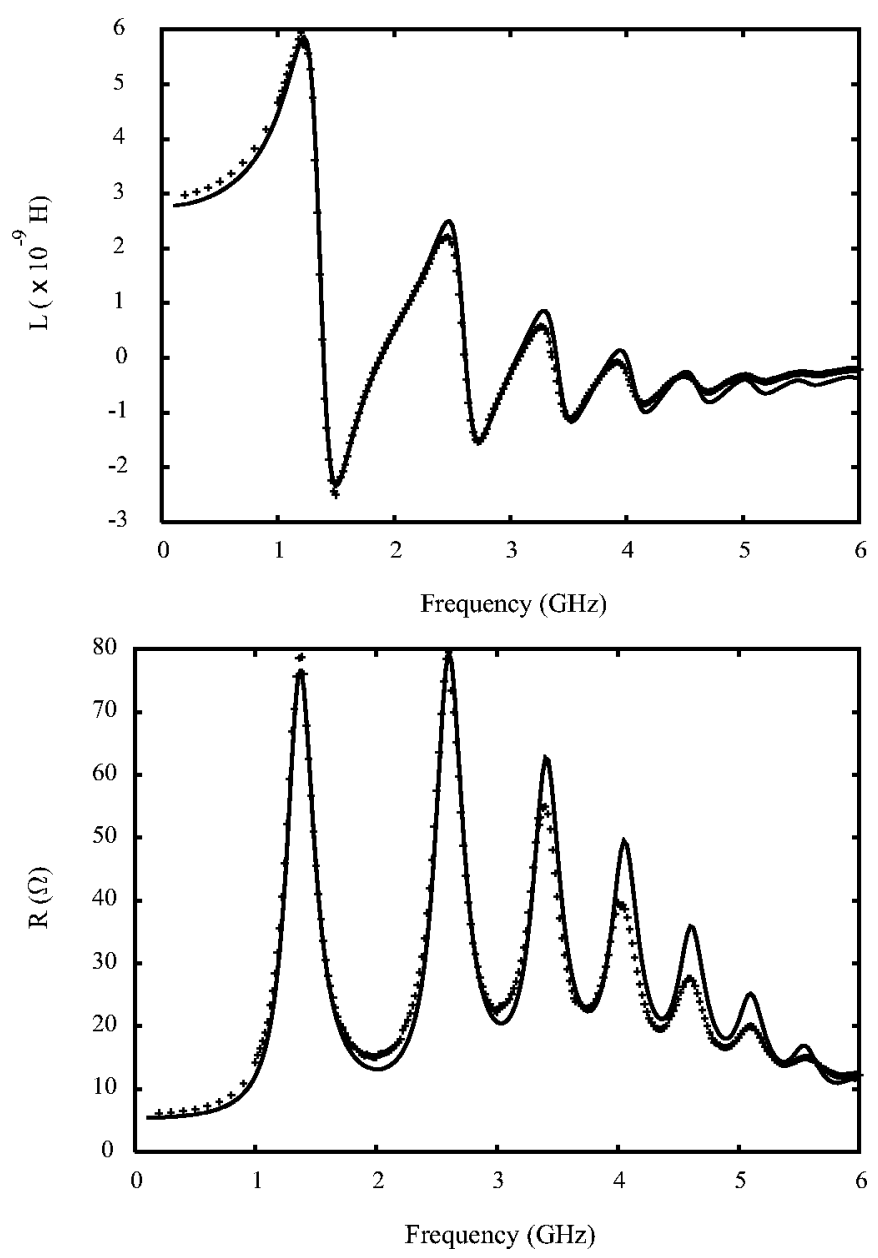

FIG. 4. Inductance $(L)$ and resistance $(R)$ of a 1-mm-long, single-turn solenoid as function of frequency. The single-turn coil is made from two parallel, $1-\mu \mathrm{m}$-thick and $10-\mu \mathrm{m}$-wide $\mathrm{Al}$ stripes. The core of the solenoid is a $100-\mu \mathrm{m}$-wide and $0.2-\mu \mathrm{m}$-thick magnetic stripe with $M_{s}=1 \mathrm{~T}, H_{a}$ $=1 \mathrm{Oe}$, and $\alpha=0.01$. The thickness of the two dielectric layers separating the coil from the stripe is $0.9 \mu \mathrm{m}$. Markers show the result of the HFSS simulation.

the same thickness. Due to the resulting symmetry of the cross section of the solenoid with respect to $y=0$, and the fact that electrical current flows in opposite directions in the top and bottom conductors, the average vertical component of the external field vanishes, i.e., $\widetilde{h}_{e, y}=0$. Thus, only $\widetilde{h}_{e, x}$ contributes to $Z_{k}$ in Eq. (18).

The resistance $R=\operatorname{Re}[Z]$ and inductance $L=\operatorname{Im}[Z] / \omega$ of the single-turn solenoid as functions of frequency are shown in Fig. 4, where one can clearly observe the even mode resonances of the magnetic stripe. Since the single-turn coil is placed around the center of the stripe at $x=0, \widetilde{h}_{e, x}(x)$ is an even function of $x$. Therefore, the odd modes of the stripe are not excited by the coil. Shifting the coil with respect to the center of the stripe changes this picture, introducing oddmode resonances (the results are not shown here).

In order to verify our results, we have also computed the inductance and resistance of the solenoid using Ansofts HFSS, a commercial full-wave electromagnetic simulator. The HFSS results, shown by markers in Fig. 4, are in good agreement with those obtained from our method, even though we use a thin-film, magnetostatic approximation. The deviation ob- 

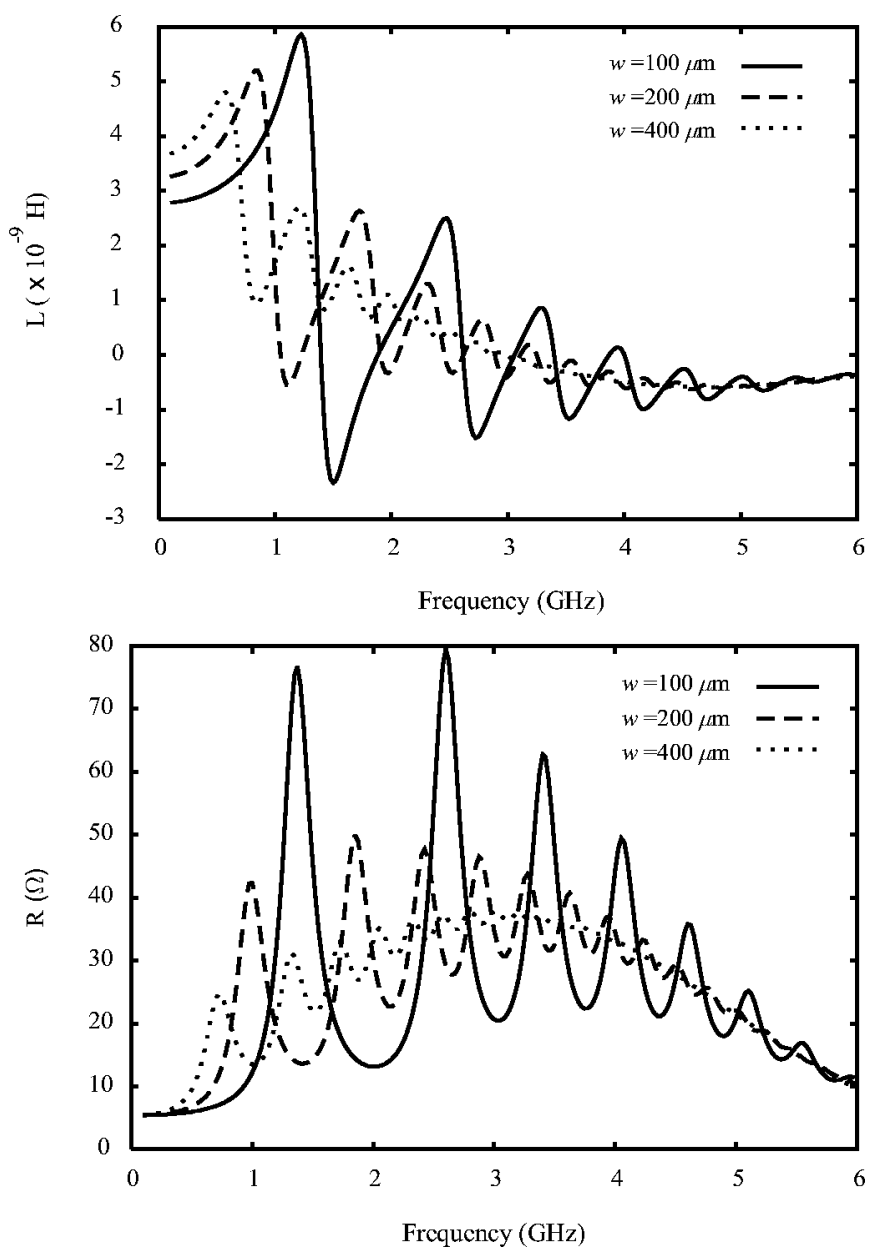

FIG. 5. Inductance $(L)$ and resistance $(R)$ of a single-turn solenoid as function of frequency for $w=100,200$, and $400 \mu \mathrm{m}$. Other parameters of the solenoid are identical to those of the solenoid in Fig. 4.

served in $R$ at frequencies above $3 \mathrm{GHz}$ is caused by modeling the metallic coil as an impressed current source, as our numerical HFSS experiments show. In reality, current distribution in a conductor reacts to the magnetic field, partially shielding it. This effect becomes stronger with increasing frequency and influences the magnetostatic modes of the stripe.

It is important to bear in mind that the multiple resonances found in the electrical characteristics of the solenoid cannot be reproduced by calculation methods based on averaged demagnetization factors. ${ }^{9-11}$ Instead, those methods predict a single resonance whose frequency, in the case of the solenoid of Fig. 4, is found to be $\sim 1.85 \mathrm{GHz}$ (details of the calculation are not given here). Furthermore, since such methods assume a uniform magnetization profile, the predicted inductance is not accurate, in particular, when the coil is placed close to the lateral edges of the stripe where the magnetization drops to small values (see Fig. 2).

The effect of increasing the width $w$ of the magnetic stripe on the impedance of the solenoid is shown in Fig. 5. Increasing $w$ causes a downward shift in the eigenvalues $\lambda_{k}$ [Eq. (8)] and reduces the separation between subsequent eigenvalues. This effect is directly reflected in the resonance spectrum of the device. The smaller zero-frequency inductance observed for wider cores is due to the reduction of the
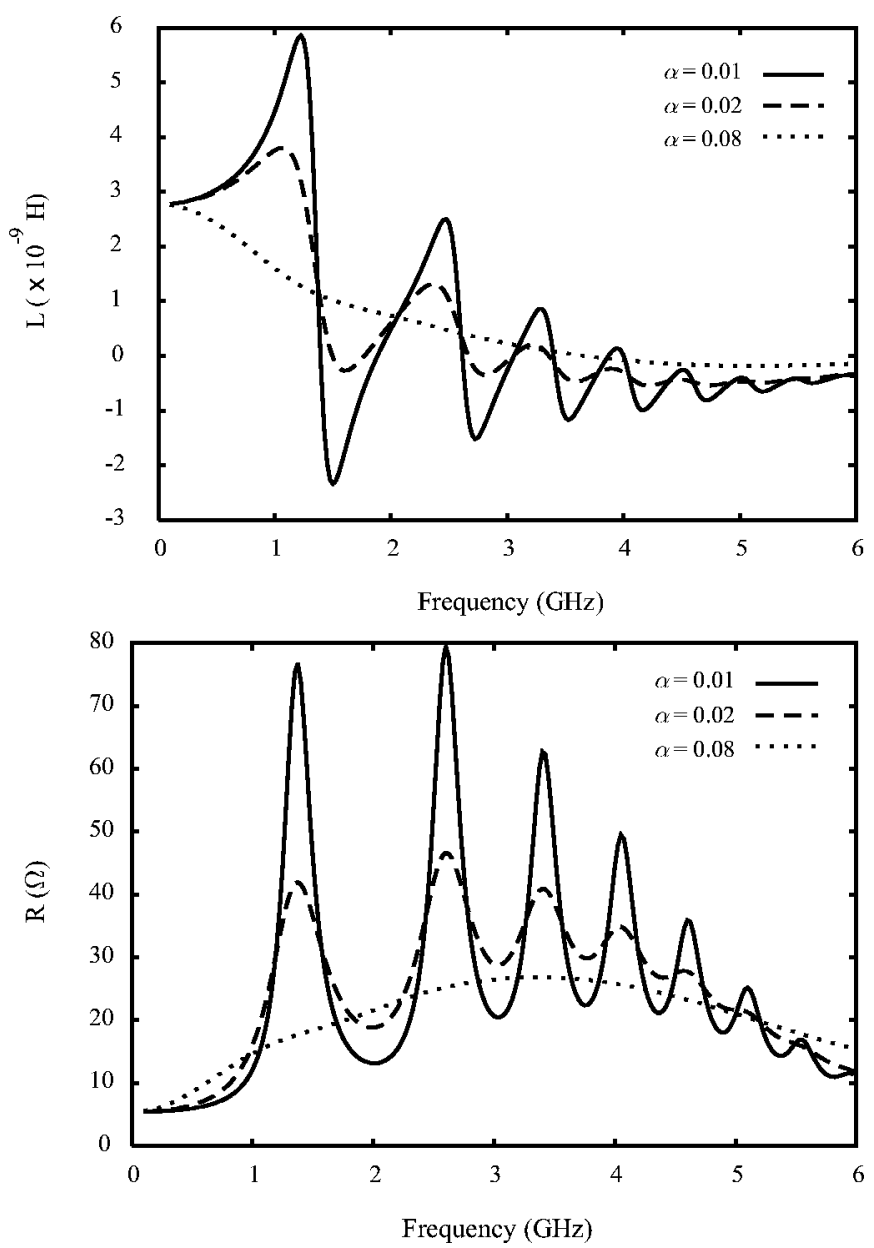

FIG. 6. Inductance $(L)$ and resistance $(R)$ of a single-turn solenoid as function of frequency for $\alpha=0.01,0.02$, and 0.08 . Other parameters of the solenoid are identical to those of the solenoid in Fig. 4.

demagnetization factors $N_{x}^{k}=1+\lambda_{k}$. This can be clearly seen by inspecting the contribution $L_{m}=\operatorname{Im}\left[Z_{m}\right] / \omega$ of the magnetic core to the zero-frequency inductance, i.e.,

$$
L_{m}(\omega \rightarrow 0)=\frac{\mu_{0} t \ell}{|I|^{2}} \sum_{k=0}^{\infty} \frac{\omega_{M}\left|\widetilde{h}_{e, x}^{k}\right|^{2}}{\omega_{H}+N_{x}^{k} \omega_{M}},
$$

where Eqs. (11), (17a), and (18) have been used.

Increasing the thickness $t$ of the magnetic stripe yields larger values of $\lambda_{k}$ and $N_{x}^{k}$, resulting in higher resonance frequencies (results are not shown). Despite larger values of $N_{x}^{k}$, however, the overall zero-frequency inductance of the device increases with thickness. This is because an increase in $N_{x}^{k}$ is overcompensated in Eq. (19) by the increase in $t$.

The individual resonances in the frequency response of a solenoid cannot be distinguished if their separation becomes smaller than their linewidth. In Fig. 6 the impedance of the single-turn solenoid is plotted for three values of the damping constant $\alpha$. By increasing $\alpha$, the detailed landscape of resonances is gradually washed out. If $\alpha$ is sufficiently large, a single, broad resonance peak is observed in the resistance, while the inductance shows a continuous drop, starting from zero frequency. This effect is, in particular, important for solenoids with a conducting magnetic core. ${ }^{7}$ The additional dissipation caused by the flow of eddy currents in the latter 
leads to a significant broadening of the resonance linewidth, smearing out the traces of magnetostatic resonances.

\section{CONCLUSION}

In this work we analyzed the rf impedance of a planar solenoid built as a planar coil enclosing a thin magnetic stripe. Employing the magnetostatic Green's function formalism, it was shown that the impedance of the solenoid can be expressed in terms of the magnetostatic eigenmodes of the stripe. This approach does not require the use of averaged demagnetization factors and naturally takes account of the effect of the magnetostatic excitations of the core on the electrical characteristics of the device. The electrical behavior of the solenoid as function of frequency depends on how the magnetic field induced by the coil is coupled to the different magnetostatic modes of the stripe, each with its specific susceptibility. The formalism developed was then used to analyze the effect of the width of the magnetic stripe and the magnetic loss on the resistance and inductance of a single-turn solenoid.

\section{ACKNOWLEDGMENTS}

We would like to thank Y. Zhuang, P. Khalili, and J. N. Burghartz for many useful discussions. This work was supported by the Foundation for Technical Sciences (STW).

${ }^{1}$ R. F. Soohoo, IEEE Trans. Magn. 15, 1803 (1979).

${ }^{2}$ M. Yamaguchi et al., J. Appl. Phys. 85, 7919 (1999).

${ }^{3}$ A. M. Crawford, D. S. Gardner, and S. X. Wang, IEEE Trans. Magn. 38 3168 (2002)

${ }^{4}$ M. Yamaguchi, M. Baba, and K.-I. Arai, IEEE Trans. Microwave Theory Tech. 49, 2331 (2001)

${ }^{5}$ V. Korenivski and R. B. van Dover, J. Appl. Phys. 82, 5247 (1997); IEEE Trans. Magn. 34, 1375 (1998)
${ }^{6}$ K. Shirakawa, H. Kurata, J. Toriu, H. Matsuki, and K. Murakami, IEEE Trans. Magn. 27, 5432 (1991).

${ }^{7}$ Y. Zhuang, M. Vroubel, B. Rejaei, and J. N. Burghartz, Tech. Dig. - Int. Electron Devices Meet. 2002, 475.

${ }^{8}$ B. Rejaei, M. Vroubel, Y. Zhuang, and J. N. Burghartz, in 4th Topical Silicon Monolithic Integrated Circuits in RF Systems Meeting Dig., 2003, 100

${ }^{9}$ D.-X. Chen, J. A. Brug, and R. B. Goldfarb, IEEE Trans. Magn. 27, 3601 (1991).

${ }^{10}$ G. Zheng, M. Pardavi-Horvath, and X. Huang, J. Appl. Phys. 79, 5742 (1996).

${ }^{11}$ A. Aharoni, J. Appl. Phys. 83, 3432 (1998)

${ }^{12}$ J. A. Osborn, Phys. Rev. 67, 351 (1945).

${ }^{13}$ R. I. Josephs and E. Schloemann, J. Appl. Phys. 36, 1579 (1965).

${ }^{14}$ L. R. Walker, Phys. Rev. 105, 390 (1957).

${ }^{15}$ C. Kittel, Phys. Rev. 110, 1295 (1958).

${ }^{16}$ C. E. Patton, Phys. Rep. 103, 251 (1984).

${ }^{17}$ A. G. Gurevich and G. A. Melkov, Magnetization Oscillations and Waves (CRC, New York, 1996).

${ }^{18}$ B. A. Kalinikos and A. N. Slavin, J. Phys. C 19, 7013 (1986).

${ }^{19}$ C. Bayer et al., Phys. Rev. B 72, 064427 (2005).

${ }^{20}$ P. H. Bryant, J. F. Smyth, S. Schultz, and D. R. Fredkin, Phys. Rev. B 47, 11255 (1993).

${ }^{21}$ K. Yu. Guslienko, S. O. Demokritov, B. Hillebrands, and A. N. Slavin, Phys. Rev. B 66, 132402 (2002)

${ }^{22}$ N. J. Moll, IEEE Trans. Microwave Theory Tech. 25, 933 (1977).

${ }^{23}$ The symmetry of the kernel $G\left(x, x^{\prime}\right)$ implies that the eigenvalues $\lambda_{k}$ and eigenfunctions $\psi_{k}(x)$ are real quantities. Furthermore, since $G\left(x, x^{\prime}\right)$ is a Hilbert-Schmidt kernel, i.e., $\int_{-w / 2}^{w / 2} \int_{-w / 2}^{w / 2} G\left(x, x^{\prime}\right) d x d x^{\prime}<\infty$, upon proper normalization one has the orthonormality condition $\int_{-w / 2}^{w / 2} \psi_{k}(x) \psi_{l}(x) d x=\delta_{k l}$, where $\delta_{k l}$ is the Kronecker delta [see F. G. Tricomi, Integral Equations (Dover, New York, 1985)]. Without proof, we also assume that the set of the eigenfunctions $\psi_{k}(x)$ is complete, so that every arbitrary function can be expanded in $\psi_{k}(x)$ on the interval $[-w / 2, w / 2]$.

${ }^{24}$ G. Counil et al. [J. Appl. Phys. 95, 5646 (2004)] find a resonance halfwidth of $2 \alpha \omega_{M}$ for an infinitely wide magnetic film. The susceptibility matrix used in that work is identical to the one in Eq. (11), with $N_{x}^{k}=0$, $N_{y}^{k}=1$. One would, therefore, expect the linewidths to be identical. We cannot point to the source of this discrepancy, as we numerically verified the relation $\Delta \omega=\alpha \omega_{M}$. 